\title{
Plasma Cell Mucositis: A Case Report of an Uncommon Benign Disease ${ }^{\dagger}$
}

\author{
Alessandro Antonelli ${ }^{1}$, Fiorella Averta ${ }^{2}$, Federica Diodati ${ }^{2}$, Danila Muraca ${ }^{2}$, Ylenia Brancaccio ${ }^{1}$, \\ Michele Davide Mignogna ${ }^{3}$ and Amerigo Giudice ${ }^{2, *}$ \\ 1 Department of Health Sciences, University Magna Graecia of Catanzaro, 88100 Catanzaro, Italy; \\ antonellicz@gmail.com (A.A.); ybrancaccio@gmail.com (Y.B.) \\ 2 Department of Health Sciences, School of Dentistry, Magna Graecia University of Catanzaro, \\ 88100 Catanzaro, Italy; fiore.averta@gmail.com (F.A.); federicadiodati@libero.it (F.D.); \\ danila.muraca@studenti.unicz.it (D.M.) \\ 3 Department of Neurosciences, Reproductive and Odontostomatological Sciences, Federico II University of \\ Naples, 80138 Naples, Italy; mignogna@unina.it \\ * Correspondence: a.giudice@unicz.it \\ † Presented at the XV National and III International Congress of the Italian Society of Oral Pathology and \\ Medicine (SIPMO), Bari, Italy, 17-19 October 2019.
}

Published: 12 December 2019

Plasma cell mucositis (PCM) is an unusual plasma cell proliferative disorder of the upper aerodigestive tract. It is a rare disease and its etiology is not yet known, it is considered a benign condition of adults and there is no correlation in literature with the development of plasma cell neoplasm. Clinical features are an intensely erythematous mucosa with papillomatous, cobblestone, nodular, or velvety surface changes. Symptoms include dysphagia, oral pain and pharyngitis [1]. Generally, PCM patients have a previous history of autoimmune or immunologically mediated disease. Despite plasma cell mucositis often involves the oral and genital mucosa, there was no genital involvement in this circumstance. We described a case of PCM involving the tongue of a 43-year-old-woman, the patient was referred to the Oral Pathology at the Faculty of Dentistry, Magna Graecia University of Catanzaro, in August 2019. Her past medical history was unremarkable. She reported a burning sensation in her mouth and local dysgeusia on the tip and the right lingual border for 5 years (Figure 1). In the first place several diagnostic hypotheses were proposed, the most of them discarded for incompatibility with blood and laboratory tests. The patient underwent an incisional biopsy under local anesthesia. The specimen was stored in a tube containing formalin $10 \%$ and sent to a laboratory for histopathological analysis. Microscopically, a large area of ulceration of the coating epithelium subtended by dense plasma cell infiltrate was observed. The final histopathological diagnosis was "Plasmacytosis of the mucous membranes with restriction for the kappa chains". This disease rarely manifests itself on the tongue, especially in young patients with no comorbidities. The management of PCM is mainly aimed at reducing the symptoms, in fact the patient was treated with systemic prednisone $50 \mathrm{mg} /$ day [2]. Many therapeutic treatments are able to stabilize but are not able to induce a complete remission. PCM is considered an uncommon benign disorder with a favorable prognosis. It is important to differentiate the PCM disease from other neoplastic conditions in order to achieve a better clinical management of the patients. 


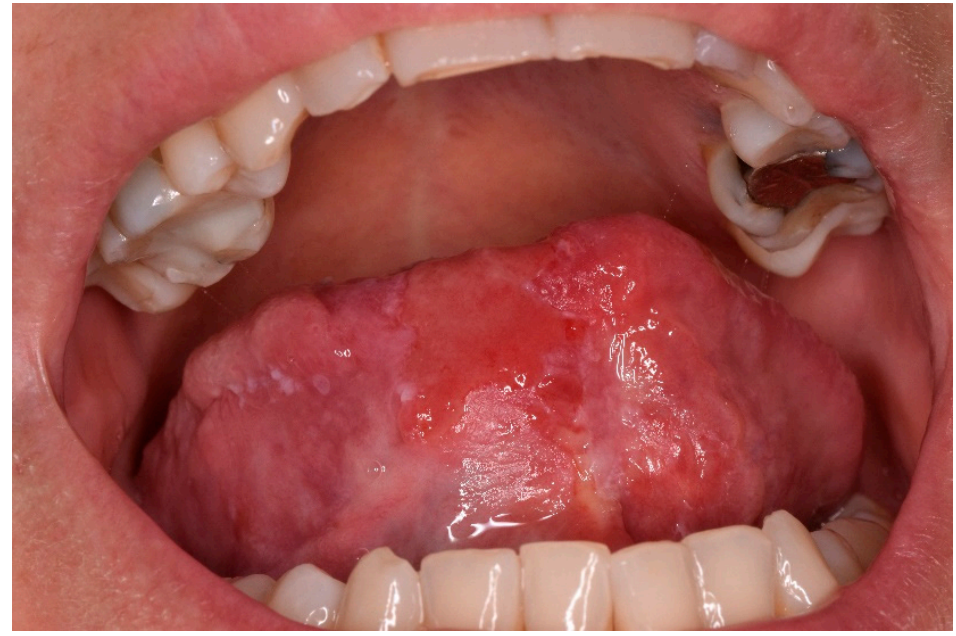

Figure 1. Ulcer localized on the tip of the tongue.

\section{References}

1. Solomon, L.W.; Wein, R.O.; Rosenwald, I.; Laver, N. Plasmacellmucositis of the oralcavity: Report of a case and review of the literature. Oral Surg. Oral Med. Oral Pathol. Oral Radiol. Endod. 2008, 106, 853-860.

2. Gasparro, R.; Adamo, D.; Masucci, M.; Sammartino, G.; Mignogna, M.D. Use of injectable platelet-rich fibrin in the treatment of plasma cell mucositis of the oral cavity refractory to corticosteroid therapy: A case report. Dermatol. Ther. 2019, 32, e13062.

(C) 2019 by the authors. Licensee MDPI, Basel, Switzerland. This article is an open access article distributed under the terms and conditions of the Creative Commons Attribution (CC BY) license (http://creativecommons.org/licenses/by/4.0/). 